06,04

\title{
Влияние концентрации примеси магния на электрические свойства кристаллов $\mathrm{LiNbO}_{3}$
}

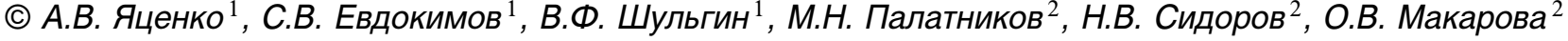 \\ ${ }^{1}$ Крымский фредеральный университет им. В.И. Вернадского, \\ Республика Крым, Симферополь, Россия, \\ ${ }^{2}$ Институт химии и технологии редких элементов и минерального сырья им. И.В. Тананаева \\ Федерального исследовательского центра „Кольский научный центр Российской академии наук“, \\ Апатиты, Россия \\ E-mail: yatsenkoav@cfuv.ru
}

Поступила в Редакцию 17 мая 2021 г.

В окончательной редакции 17 мая 2021 г.

Принята к публикации 21 мая 2021 г.

С использованием результатов исследования электрической проводимости в диапазоне температур $\sim 295-460 \mathrm{~K}$ и спектров ИК-поглощения серии кристаллов $\mathrm{LiNbO}_{3}: \mathrm{Mg}$ показано, что по мере увеличения содержания $\mathrm{Mg}$ в образце резко уменьшается подвижность ионов $\mathrm{H}^{+}$при перемещении вдоль полярного направления. Наиболее ярко этот эффект проявляется в образцах кристаллов $\mathrm{LiNbO}_{3}: \mathrm{Mg}$ с высоким (сверхпороговым) содержанием примеси.

Ключевые слова: сегнетоэлектрики, ниобат лития, электропроводность, подвижность, легирование, примеси.

DOI: $10.21883 /$ FTT.2021.10.51407.118

\section{1. Ввеедние}

Ниобат лития $\mathrm{LiNbO}_{3}$ и танталат лития $\mathrm{LiTaO}_{3}$ являются одними из наиболее важных сегнетоэлектрических материалов для применения в акустоэлектронике и оптоэлектронике благодаря присущему им комплексу физических свойств [1]. Хорошо известно, что легирование кристаллов $\mathrm{LiNbO}_{3}$ магнием сильно влияет на многие физические свойства таких кристаллов [2]. В частности, наличие ионов $\mathrm{Mg}^{2+}$ с концентрацией, выше пороговой ( $\sim 4.8 \mathrm{~mol} \% \mathrm{MgO}$ в кристалле) приводит к резкому подавлению так называемого фоторефрактивного эффекта (фотоиндуцированное изменение разности показателей преломления) [3], а в плане электрических свойств к существенному уменьшению значения коэрцитивного поля по сравнению с номинально беспримесным кристаллом $\mathrm{LiNbO}_{3}$ конгруэнтного состава (CLN congruent lithium niobate) $\mathrm{Li} / \mathrm{Nb}=0.946$ [4].

Влияние примеси магния на удельную электрическую проводимость $\sigma$ кристаллов $\mathrm{LiNbO}_{3}$, впервые рассматривалось в [5]. Исследовалась серия образцов, включая CLN и образец, выращенный из шихты, содержащей $5.0 \mathrm{~mol} \% \mathrm{MgO}$, которая прошла дополнительную окислительную обработку. Согласно [5] в температурном диапазоне $\sim 460-590 \mathrm{~K}$ примесь $\mathrm{Mg}$ не влияет на значение энергии активации $E_{a}$, которая составляла величину $\sim 1.21 \mathrm{eV}$.

Электрическая проводимость кристаллов $\mathrm{LiNbO}_{3}: \mathrm{Mg}$, выращенных из расплава с разным соотношением $\mathrm{Li} / \mathrm{Nb}$ и вариацией содержания $\mathrm{Mg}$ в диапазоне температур 613-733 К была исследована в [6]. Отмечено, что по мере увеличения содержания магния значение $E_{a}$ увеличивается, а удельная электрическая проводимость $\sigma$ уменьшается. Такая же тенденция отмечена и в [7].

В [8] представлены результаты исследования серии кристаллов $\mathrm{LiNbO}_{3}: \mathrm{Mg}$ содержащих до $10 \mathrm{~mol} \%, \mathrm{MgO}$ при $T=(297-476) \mathrm{K}$. Отмечалось, что значение $E_{a}$ лежит в интервале $(1.1-1.5) \mathrm{eV}$, однако четкой концентрационной закономерности в этих данных не прослеживается. Согласно [9] в диапазоне $(362-465) \mathrm{K}$ значения $E_{a}$ для CLN и образца $\mathrm{LiNbO}_{3}: \mathrm{Mg}$ с содержанием $5 \mathrm{~mol} \% \mathrm{MgO}$ практически одинаковы и составляют $\sim 1.04 \mathrm{eV}$.

Для кристаллов $\mathrm{LiNbO}_{3}: \mathrm{Mg}(\sim 5.0 \mathrm{~mol} \% \mathrm{MgO}$ в расплаве) сообщались следующие значения $\sigma$ при комнатной температуре $\left(\sigma_{d}\right)_{r t}: 5.5 \cdot 10^{-16}(\Omega \cdot \mathrm{cm})^{-1}[10]$ и $1.9 \cdot 10^{-15}(\Omega \cdot \mathrm{cm})^{-1} \quad[11]$, что существенно больше, чем у номинально беспримесных CLN при $T=300 \mathrm{~K}[12,13]$. Это вполне согласуется с тенденцией к увеличению $\left(\sigma_{d}\right)_{r t}$ в кристаллах $\mathrm{LiNbO}_{3}: \mathrm{Mg}$ по сравнению с CLN [14,15], но противоречат данным, полученным при более высоких температурах [6,7]. При этом отмечалось, что проводимость кристаллов $\mathrm{LiNbO}_{3}: \mathrm{Mg}$ изотропна [16]. Однако в [17] зафиксирована существенная анизотропия электрической проводимости образцов $\mathrm{LiNbO}_{3}: \mathrm{Mg}$ при приложении внешнего поля вдоль полярного и неполярных направлений, но при одинаковом значении $E_{a}$.

В публикациях [6-17] для анализа удельной электрической проводимости $\sigma(T)$ применялся закон Аррениуca, учитывающий обобщенный ионный и - в некоторых случаях - электронный вклад в проводимость. Соот- 
Таблица 1. Электрические свойства кристаллов $\mathrm{LiNbO}_{3}$ различного химического состава при $T=400 \mathrm{~K}$

\begin{tabular}{c|c|c|c|c}
\hline $\begin{array}{c}\text { Содержание } \\
\mathrm{MgO}, \mathrm{mol} \%\end{array}$ & Ориентация & $E_{a}, \mathrm{eV}$ & $\begin{array}{c}\sigma_{400 \mathrm{~K},} \\
(\Omega \cdot \mathrm{cm})^{-1}\end{array}$ & Ссылка \\
\hline $\mathrm{CLN}$ & $z$-сре3 & $1.05 \pm 0.02$ & $\approx 1.03 \cdot 10^{-13}$ & {$[9]$} \\
\hline 5.0 & $z$-сре3 & $1.03 \pm 0.01$ & $\approx 7.5 \cdot 10^{-14}$ & {$[9]$} \\
\hline 2.0 & $z$-сре3 & $1.07 \pm 0.01$ & $\approx 2.1 \cdot 10^{-14}$ & {$[12]$} \\
\hline $\mathrm{CLN}$ & $z$-сре3 & 1.0 & $\approx 4.1 \cdot 10^{-14}$ & {$[17]$} \\
\hline 5.0 & $z$-сре3 & 1.2 & $\approx 1 \cdot 10^{-14}$ & {$[17]$} \\
\hline 5.0 & $y$-сре3 & 1.2 & $\approx 7.5 \cdot 10^{-15}$ & {$[17]$} \\
\hline 5.0 & $x$-сре3 & 1.2 & $\approx 5 \cdot 10^{-15}$ & {$[17]$}
\end{tabular}

ветствующее выражение, описывающее температурную зависимость $\sigma(T)$ имеет следующий вид:

$$
\sigma(T)=\frac{C_{i}}{T} \exp \left(-\frac{E_{a}}{k_{0} T}\right)+C_{e} \exp \left(-\frac{E_{e}}{k_{0} T}\right)
$$

где $k_{0}-$ постоянная Больцмана, $T-$ температура, $E_{a}$ и $E_{e}$ - энергии активации ионов и электронной проводимости соответственно, $C_{i}$ и $C_{e}-$ коэффициенты, которые зависят от объемной концентрации и подвижности соответствующего типа носителей. Некоторые известные данные о электрической проводимости кристаллов $\mathrm{LiNbO}_{3}: \mathrm{Mg}$ при $T=400 \mathrm{~K}$ приведены в табл. 1.

Из сделанного обзора следует, что при $T>373 \mathrm{~K}$ значения $E_{a}$ кристаллов $\mathrm{LiNbO}_{3}: \mathrm{Mg}$ с критическим содержанием магния могут лежать в диапазоне $\sim 1.0-1.2 \mathrm{eV}$. Из этой закономерности выпадают только данные, приведенные в работе [8]. Имеющиеся расхождения в результатах измерения энергии активации могут объясняться термической предисторией образцов, исследованных разными авторами - в частности проведением или не проведением послеростовой окислительной термохимической обработки кристаллов.

Во всех известных к настоящему времени работах за исключением [12] температурная зависимость про- водимости кристаллов $\mathrm{LiNbO}_{3}: \mathrm{Mg}$ вблизи комнатной температуры не изучалась. При этом следует учитывать, что реальное практическое использование нефоторефрактивного кристалла $\mathrm{LiNbO}_{3}: \mathrm{Mg}$ обычно имеет место именно при $T \sim 300 \mathrm{~K}$.

Поэтому основной целью работы является исследование темновой электрической проводимости серии кристаллов $\mathrm{LiNbO}_{3}: \mathrm{Mg}$ выращенных из шихты конгруэнтного состава с добавлением примеси магния (прямое легирование) и анализ влияния содержания $\mathrm{Mg}$ на их электрическую проводимость и подвижность носителей.

\section{2. Образцы и методика эксперимента}

Bce образцы $\mathrm{LiNbO}_{3}: \mathrm{Mg}$, исследованные в настоящей работе, были выращены методом Чохральского из шихты конгруэнтного состава с добавлением в исходный расплав разного количества $\mathrm{MgO}$ (прямое легирование) и прошли процедуру монодоменизации. Ни один из образцов $\mathrm{LiNbO}_{3}: \mathrm{Mg}$ не подвергался каким либо дополнительным послеростовым термохимическим обработкам. Состав исследованных образцов приведен в табл. 2. Образцы № 1,3 и 4 были синтезированы в ИХТРЭМС (Апатиты, Россия), образец № 2 - в Институте физики твердого тела и оптики (The Institute for Solid State Physics and Optics, Budapest, Hungary). Образец № 5 был выращен в НПО „Карат“ (Львов, Украина).

Измерения электрической проводимости образцов по постоянному току проводились двухточечным методом - c нанесением на полярные поверхности кристалла электродов из индий-галлиевой эвтектики. Методика предварительной подготовки образцов и проведения измерений подробно рассмотрена в работе [18].

Объемная концентрация $\mathrm{OH}^{-}$групп в исследуемых образцах $\left(c_{\mathrm{OH}}\right)$ установлена из анализа спектров ИК поглощения в области $(3400-3600) \mathrm{cm}^{-1}$, полученных на FTIR спектрометре ФСМ-2202. ИК-измерения выполнялись на образцах $z$-среза при неполяризованном излучении и комнатной температуре, а определение $c_{\mathrm{OH}^{-}}$проводилось по методике, предложенной в [19].

Таблица 2. Концентрация дефектов в исследуемых кристаллах $\mathrm{LiNbO}_{3}$ при комнатной температуре

\begin{tabular}{|c|c|c|c|c|c|}
\hline Образец & $\begin{array}{l}\text { Содержание } \\
\mathrm{MgO}, \mathrm{mol} \%\end{array}$ & $\begin{array}{c}c_{\mathrm{Nb}_{\mathrm{Li}}}, \\
10^{19} \mathrm{~cm}^{-3}\end{array}$ & $\begin{array}{c}c_{\mathrm{V}_{\mathrm{Li}}}, \\
10^{19} \mathrm{~cm}^{-3}\end{array}$ & $\begin{array}{l}A_{\text {int }}, \\
\mathrm{cm}^{2}\end{array}$ & $\begin{array}{c}c_{\mathrm{OH}^{-}}, \\
10^{18} \mathrm{~cm}^{-3}\end{array}$ \\
\hline 1 & CLN & $\sim 20$ & $\sim 80$ & 42 & $2.4 \pm 0.4$ \\
\hline 2 & $\sim 2.0$ (в расплаве $)$ & $\sim 11.5$ & $\sim 86$ & 44 & $2.5 \pm 0.4$ \\
\hline 3 & 3.1 (в кристалле) & $\sim 7.6$ & $\sim 90$ & 30 & $1.7 \pm 0.3$ \\
\hline 4 & 4.7 (в кристалле) & $\sim 0.4$ & $\sim 94$ & 25 & $1.4 \pm 0.2$ \\
\hline 5 & 7.0 (в кристалле) & $\sim 0$ & $\sim 97$ & 42 & $2.4 \pm 0.4$ \\
\hline
\end{tabular}




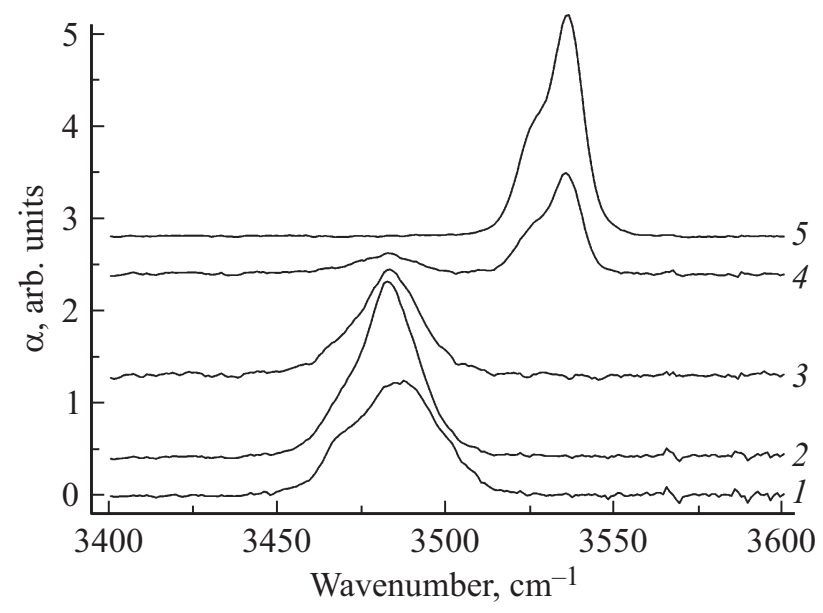

Рис. 1. Вид спектров ИК-поглощения в области валентных колебаний $\mathrm{OH}^{-}$групп для исследованных кристаллов $\mathrm{LiNbO}_{3}$ № 1-5. Номер зависимости на рисунке соответствует номеру образца в табл. 2-5.

\section{3. Экспериментальные результаты}

Спектральное распределение ИК поглощения для данной серии кристаллов $\mathrm{LiNbO}_{3}: \mathrm{Mg}$ и кристалла CLN представлено на рис. 1. Эти результаты достаточно хорошо согласуются с известными результатами для кристаллов $\mathrm{LiNbO}_{3}$ близкого состава [20-22]. Наглядно видно, что полоса поглощения при значении волнового числа $\bar{v} \approx 3533 \mathrm{~cm}^{-1}$ присутствует только у образцов № 4 и 5. Хорошо известно, что эта полоса отвечает валентным колебаниям $\mathrm{OH}^{-}$групп ассоциированных с дефектом замещения $\mathrm{Mg}_{\mathrm{Nb}}$ [21,22], поэтому ее наличие свидетельствует о отсутствии антиструктурных ионов $\mathrm{Nb}_{\mathrm{Li}}$ в образце № 5. Результаты расчета площади ИК кривой поглощения $A_{\text {int }}$ и концентрации $\mathrm{OH}^{-}$групп в конкретных образцах приведены в табл. 2. Там же приводятся результаты вычисления объемной концентрации вакансий лития $c_{\mathrm{V}_{\mathrm{Li}}}$ и антиструктурных ионов $\mathrm{Nb}_{\mathrm{Li}} c_{\mathrm{Nb}_{\mathrm{Li}}}$. Расчеты концентрации дефектов выполнялись по данным, приведенным в работе [23].

Температурные зависимости $\sigma(T)$ исследованной серии кристаллов $\mathrm{LiNbO}_{3}$ при приложении внешнего элек-

Таблица 3. Параметры, описывающие зависимость $\sigma(T)$ выражением (1)

\begin{tabular}{c|c|c|c|c}
\hline Образец & $\sigma_{400 \mathrm{~K}},(\Omega \cdot \mathrm{cm})^{-1}$ & $E_{a}, \mathrm{eV}$ & $E_{e}, \mathrm{eV}$ & $\delta, \%$ \\
\hline 1 & $7.16 \cdot 10^{-14}$ & $1.05 \pm 0.01$ & $0.29 \pm 0.02$ & 1.1 \\
\hline 2 & $2.08 \cdot 10^{-14}$ & $1.10 \pm 0.01$ & $0.36 \pm 0.03$ & 1.2 \\
\hline 3 & $1.10 \cdot 10^{-14}$ & $1.19 \pm 0.01$ & $0.29 \pm 0.02$ & 1.2 \\
\hline 4 & $8.9 \cdot 10^{-15}$ & $1.23 \pm 0.01$ & $0.34 \pm 0.03$ & 1.1 \\
\hline 5 & $5.31 \cdot 10^{-15}$ & $1.26 \pm 0.01$ & $0.31 \pm 0.03$ & 1.6
\end{tabular}

трического поля вдоль полярной оси образца представлены на рис. 2 и 3. Наглядно видно, что по мере увеличения содержания магния в кристалле удельная электрическая проводимость в области температур $350-450 \mathrm{~K}$ уменьшается, что сопровождается увеличением усредненного значения энергии активации $E_{a}$, отвечающей за ионный вклад в проводимость. Значения $E_{a}$ и $E_{e}$ полученные при оптимальной аппроксимации результатов выражением (1) приведены в табл. 3. В этой же таблице приводятся значения среднеквадратичного отклонения $\delta$ экспериментальных результатов от аппроксимирующей кривой, которое вычислялось следующим образом:

$$
\delta=\sqrt{\frac{\sum_{i=1}^{n}\left(1-s_{i} \sigma_{i}^{-1}\right)^{2}}{n(n-1)}},
$$

где $n$ - количество экспериментальных точек, $\sigma_{i}$ и $s_{i}$ экспериментальное и вычисленное значение удельной

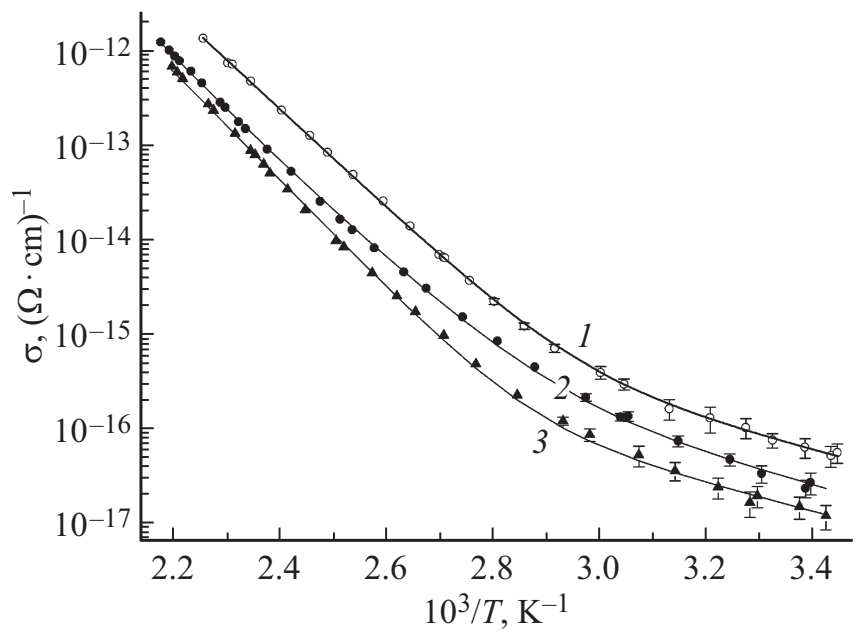

Рис. 2. Зависимости $\sigma(T)$ при приложении внешнего электрического поля вдоль оси $z$ для образцов $\mathrm{LiNbO}_{3}$ №№ 1-3.

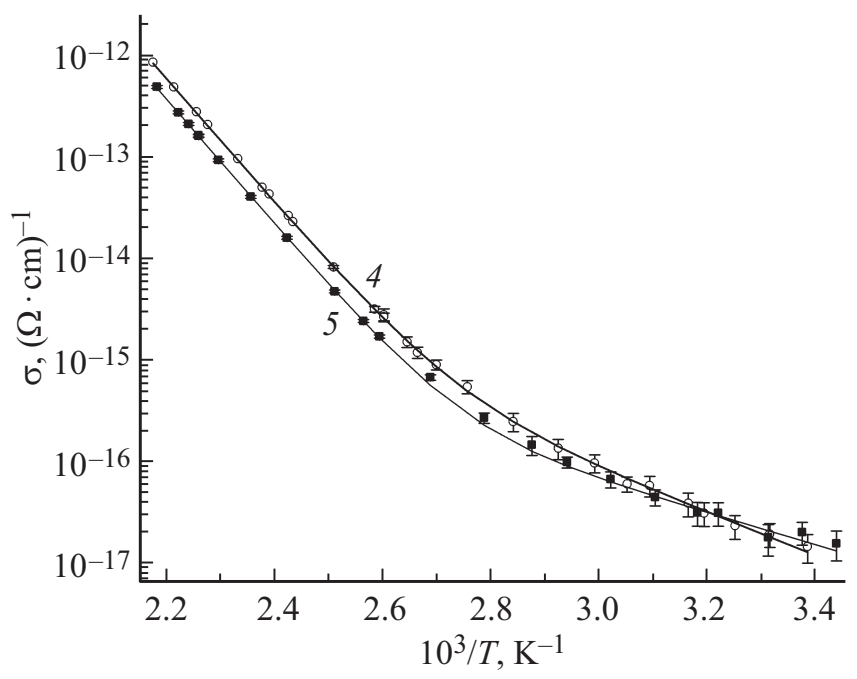

Рис. 3. Зависимости $\sigma(T)$ при приложении внешнего электрического поля вдоль оси $z$ для образцов $\mathrm{LiNbO}_{3}: \mathrm{Mg}$ № 4-5. 
Таблица 4. Результаты оптимальной аппроксимации зависимости $\sigma(T)$ выражением (3) при фиксированных значениях энергий активации

\begin{tabular}{c|c|c|c|c}
\hline Образец & $A_{1},(\Omega \cdot \mathrm{cm})^{-1} \cdot \mathrm{K}$ & $A_{2},(\Omega \cdot \mathrm{cm})^{-1} \cdot \mathrm{K}$ & $A_{3},(\Omega \cdot \mathrm{cm})^{-1} \cdot \mathrm{K}$ & $\delta, \%$ \\
\hline 1 & $(207 \pm 24) \cdot 10^{-3}$ & $(230 \pm 6) \cdot 10^{-6}$ & $(56 \pm 5) \cdot 10^{-13}$ & 1.2 \\
\hline 2 & $(98 \pm 3) \cdot 10^{-3}$ & $(61 \pm 2) \cdot 10^{-6}$ & $(25 \pm 3) \cdot 10^{-13}$ & 1.7 \\
\hline 3 & $(105 \pm 3) \cdot 10^{-3}$ & $(26 \pm 1) \cdot 10^{-6}$ & $(13 \pm 2) \cdot 10^{-13}$ & 1.2 \\
\hline 4 & $(113 \pm 3) \cdot 10^{-3}$ & $(16 \pm 0.5) \cdot 10^{-6}$ & $(13 \pm 2) \cdot 10^{-13}$ & 1.5 \\
\hline 5 & $74 \pm 2$ & $(8.5 \pm 0.4) \cdot 10^{-6}$ & $(15 \pm 2) \cdot 10^{-13}$ & 1.6
\end{tabular}

проводимости в $i$-й точке. Данные табл. 3. однозначно свидетельствуют о увеличении значения $E_{a}$ и уменьшении проводимости при $T=400 \mathrm{~K}\left(\sigma_{400 \mathrm{~K}}\right)$ по мере увеличения содержания $\mathrm{Mg}$ в образце. Это целиком подтверждает выводы, сделанные ранее в работах [6,7].

\section{4. Обсуждение результатов}

Недавно было показано, что электрическая проводимость кристаллов $\mathrm{LiNbO}_{3}$ различного состава, имеющих несколько разных значений энергии активации $E_{a}$, может быть корректно описана в предположении, что в процессе переноса заряда участвуют как электроны, так и одновременно два типа подвижных ионов с разными энергиями активации [24]. Уравнение, описывающее температурную зависимость удельной электрической проводимости $\sigma(T)$, при этом приобретает следующий вид:

$$
\begin{aligned}
\sigma(T)= & \frac{A_{1}}{T} \exp \left(-\frac{E_{1}}{k_{0} T}\right)+\frac{A_{2}}{T} \exp \left(-\frac{E_{2}}{k_{0} T}\right) \\
& +A_{3} \exp \left(-\frac{E_{3}}{k_{0} T}\right),
\end{aligned}
$$

где $A_{1}, A_{2}, A_{3}$ - соответствующие коэффициенты пропорциональности, $E_{1}$ и $E_{2}-$ энергии активации ионов первого и второго сорта, $E_{3}-$ энергия активации электронной проводимости. Было установлено [24], что максимальная точность аппроксимации обеспечивается при следующей комбинации энергий активации: $E_{1}=1.33 \mathrm{eV}, E_{2}=1.03 \mathrm{eV}$ и $E_{3}=0.29 \mathrm{eV}$. Причем точность этого описания не хуже, чем описание выражением (1), которое предполагает один тип ионной проводимости. Эти значения отвечают энергиям активации подвижности ионов $\mathrm{Li}^{+}$[25], ионов $\mathrm{H}^{+}$и поляронной проводимости [26] соответственно. Использование уравнения (3) при фиксированных значениях энергий активации дает возможность сравнить вклад носителей каждого типа в проводимость исследуемых кристаллов и, следовательно, оценить изменение подвижности этих носителей.

Значения коэффициентов $A_{1}, A_{2}$ и $A_{3}$, полученные из оптимальной аппроксимации экспериментальных дан-

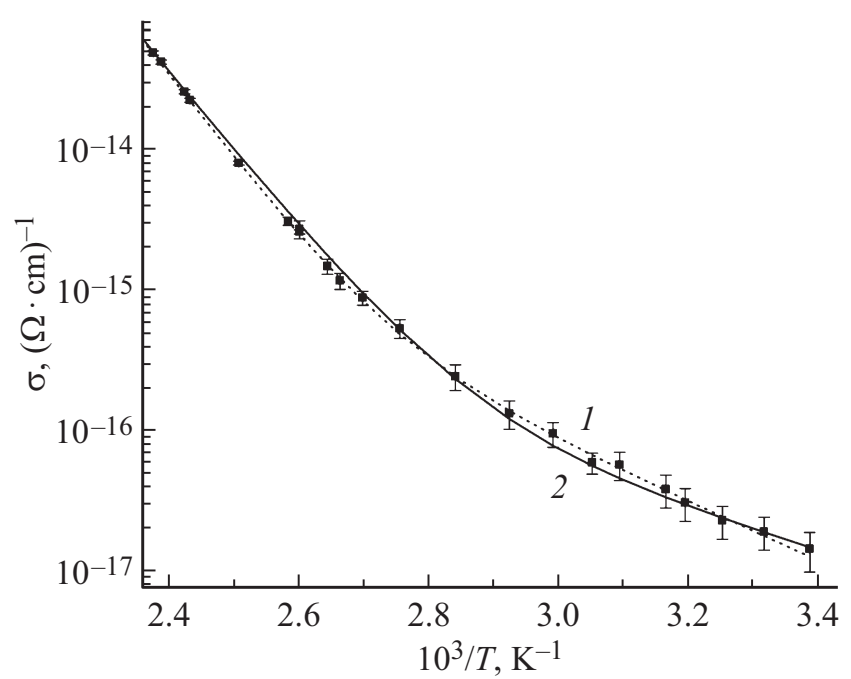

Рис. 4. Аппроксимация экспериментальных данных для образца $\mathrm{LiNbO}_{3}$ : Mg № 4: $(1)$ - выражением (1); (2) - выражением (3).

ных для всех исследованных выше образцов выражением (3) при $E_{1}=1.33 \mathrm{eV}, E_{2}=1.03 \mathrm{eV}$ и $E_{3}=0.29 \mathrm{eV}$ представлены в табл. 4. При сопоставлении среднеквадратичных отклонений $\delta$, приводящихся в табл. 3 и 4 для соответствующих образцов № 1-5, видно, что применение выражения (3) вполне обосновано, так как доминирующий вклад в небольшое увеличение $\delta$ (образцы № 2 и 4) дает несоответствие значений $E_{3}$ и $E_{e}$. На рис. 4 приведены результаты оптимальной аппроксимации данных для образца № 4 (низкотемпературная часть) выражениями (1) и (3) - видно, что обе зависимости лежат практически в пределах ошибок эксперимента.

Классическое выражение, связывающее удельную проводимость кристаллов по постоянному току $\sigma$ с подвижностью ионов $\mu$, имеет следующий вид:

$$
\sigma=q n \mu=\frac{q n \mu_{0}}{T} \exp \left(-\frac{E_{a}}{k_{0} T}\right),
$$

где $q$ - заряд иона, $n$ - объемная концентрация вакантных позиций ионов $\mathrm{Li}^{+}$, либо объемная концентрация 
Таблица 5. Предэкспоненциальные множители $\mu_{\mathrm{Li}}$ и $\mu_{\mathrm{H}}$ в кристалах $\mathrm{LiNbO}_{3}$ различного химического состава

\begin{tabular}{c|c|c}
\hline Образец & $\mu_{\mathrm{Li}}, \mathrm{K} \cdot \mathrm{cm}^{2} \cdot \mathrm{V}^{-1} \cdot \mathrm{s}^{-1}$ & $\mu_{\mathrm{H}}, \mathrm{K} \cdot \mathrm{cm}^{2} \cdot \mathrm{V}^{-1} \cdot \mathrm{s}^{-1}$ \\
\hline 1 & $(16.2 \pm 1.2) \cdot 10^{-4}$ & $(598 \pm 65) \cdot 10^{-6}$ \\
\hline 2 & $(7.1 \pm 0.2) \cdot 10^{-4}$ & $(152 \pm 17) \cdot 10^{-6}$ \\
\hline 3 & $(7.3 \pm 0.2) \cdot 10^{-4}$ & $(95 \pm 9) \cdot 10^{-6}$ \\
\hline 4 & $(7.5 \pm 0.2) \cdot 10^{-4}$ & $(71 \pm 9) \cdot 10^{-6}$ \\
\hline 5 & $(4.8 \pm 0.2) \cdot 10^{-4}$ & $(22 \pm 4) \cdot 10^{-6}$
\end{tabular}

ионов $\mathrm{H}^{+} ; \mu_{0}-$ предэкспоненциальный множитель, $E_{a}$ - энергия активации. Из сопоставления (3) и (4) получим выражения, позволяющие вычислить предэкспоненциальные множители для ионов $\mathrm{Li}^{+}$и $\mathrm{H}^{+}$при их миграции вдоль полярного направления

$$
\mu_{\mathrm{Li}}=\frac{A_{1}}{|e| c_{\mathrm{V}_{\mathrm{Li}}}} \quad \text { и } \quad \mu_{\mathrm{H}}=\frac{A_{2}}{|e| c_{\mathrm{OH}^{-}}},
$$

где $|e|$ - модуль заряда электрона. Результаты расчета $\mu_{\mathrm{Li}}$ и $\mu_{\mathrm{H}}$ приведены в табл. 5 .

Представленные в табл. 5 результаты свидетельствуют о том, что подвижность ионов $\mathrm{Li}^{+}$и $\mathrm{H}^{+}$вдоль полярной оси кристалла уменьшается при введении в кристалл нефоторефрактивных ионов $\mathrm{Mg}^{2+}$. Этот факт полностью согласуется с наблюдавшимся ранее уменьшением коэффициента диффузии дейтерия в кристаллах $\mathrm{LiNbO}_{3}: \mathrm{Mg}(8 \mathrm{~mol} \% \mathrm{MgO})$ по сравнению с образцом кристалла CLN [27].

Быстрое уменьшение подвижности ионов $\mathrm{H}^{+}$по мере увеличения содержания $\mathrm{Mg}$ в образцах может быть связано с возникновением дефектных комплексов типа $\left(4 \mathrm{Mg}_{\mathrm{Li}}^{2+}+4 \mathrm{~V}_{\mathrm{Li}}\right)$ [28]. Эти комплексы из-за своей размерности искажают локальное внутрикристаллическое электрическое поле в большем объеме кристалла, чем дефектные кластеры $\left(\mathrm{Nb}_{\mathrm{Li}}^{5+}+4 \mathrm{~V}_{\mathrm{Li}}\right)$, характерные для кристаллов CLN. Эти искажения, по-видимому, приводят к таким изменениям потенциального рельефа, которые уменьшают вероятность переноса ионов $\mathrm{H}^{+}$в полярном направлении.

Таким образом, значительный разброс известных значений $E_{a}$ для кристаллов $\mathrm{LiNbO}_{3}: \mathrm{Mg}$ с различным содержанием магния [5-7] может объясняться как различной концентрацией ОН-групп, так и влиянием состава кристалла на подвижность ионов $\mathrm{H}^{+}$и, соответственно, на их вклад в ионную компоненту проводимости.

\section{5. Заключение}

Установлено, что увеличение концентрации $\mathrm{Mg}$ в составе кристаллов $\mathrm{LiNbO}_{3}: \mathrm{Mg}$ сопровождается прогрессируюшим уменьшением подвижности примесных ионов $\mathrm{H}^{+}$и, таким образом, значительно уменьшает ионный вклад в электрическую проводимость при той же объемной концентрации ионов $\mathrm{H}^{+}$.

Из этого можно сделать важный практический вывод о том, что время релаксации объемных фазовых голограмм, записанных в кристалле двойного легирования $\mathrm{LiNbO}_{3}: \mathrm{Mg}, \mathrm{Fe}$ с использованием методики термической фиксации [3] должно быть существенно больше, чем в образцах $\mathrm{LiNbO}_{3}: \mathrm{Fe}$ при такой же концентрации как железа, так и протонов. Подробному исследованию анизотропии электрической проводимости и, соответственно, анизотропии подвижности ионов в кристаллов $\mathrm{LiNbO}_{3}: \mathrm{Mg}$ будет посвящена отдельная публикация.

\section{Финансирование работы}

Работа была выполнена в рамках государственного задания Министерства науки и высшего образования Российской Федерации (научная тема № 0186-2021-0022, регистрационный номер АAАA-A18-118022190125-2).

\section{Конфликт интересов}

Авторы заявляют об отсутствии конфликта интересов.

\section{Список литературы}

[1] L. Arizmendi. Phys. Status Solidi A 201, 253 (2004). DOI: $10.1002 /$ pssa.200303911.

[2] B.C. Grabmaier, W. Wersing, W. Koestler. J. Cryst. Growth. 110, 339 (1991). DOI: 10.1016/0022-0248(91)90269-B.

[3] T.R. Volk, M. Wöhlecke. Lithium Niobate. Defects, Photorefraction and Ferroelectric Switching. Springer-Verlag, Berlin (2008).

[4] P. Sen, N. Sisodia, R. Choubey, S. Kar, K. Bartwal. J. Nonlinear Opt. Phys. \& Mater. 17, 175 (2008). DOI: $10.1142 / \mathrm{S} 0218863508004068$.

[5] R. Gerson, J.F. Kirchhoff, L.E. Halliburton, D.A. Bryan. J. Appl. Phys. 60, 3553 (1986). DOI: 10.1063/1.337611.

[6] L. Kovács, I. Földvári, K. Polgár. Acta Phys. Hungarica 61, 2, 223 (1987). DOI: 10.1007/BF03155897.

[7] W. Rossner, B.C. Grabmaier, W. Wersing. Ferroelectrics 93, 57 (1989). DOI: 10.1080/00150198908017324.

[8] I.W. Kim, S.W. Kim, Y.H. Hwang, B.M. Jin, S.C. Kim, V.F. Pichugin, T.S. Frangulian, V.F. Stoliarenko. Ferroelectrics 261, 263 (2001). DOI: 10.1080/00150190108216295.

[9] N. Meyer, G.F. Nataf, T. Granzow. J. Appl. Phys. 115, 244102 (2014). DOI: $10.1063 / 1.4905021$.

[10] H. Wang, G. Shi, Z. Wu. Phys. Status Solidi A. 89, K212 (1985). DOI: $10.1002 /$ pssa.2210890256.

[11] M. Wengler, U. Heinemeyer, E. Soergel, K. Buse. J. Appl. Phys. 98, 6, 064104 (2005). DOI: 10.1063/1.2058184.

[12] S.V. Yevdokimov, A.V. Yatsenko. Phys. Solid State 48, 336 (2006). DOI: $10.1134 / \mathrm{S} 1063783406020247$

[13] A.V. Yatsenko, A.S. Pritulenko, S.V. Yevdokimov, D.Yu. Sugak, I.I. Solskii. Solid State Phenom. 200, 193 (2013). DOI: 10.4028/www.scientific.net/SSP.200.193.

[14] K. Mizuuchi, A. Morikawa, T. Sugita, K. Yamamoto. Electron. lett. 40 (13) 819 (2004). DOI: 10.1049/el:20040515. 
[15] V. Ya. Shur, I.S. Baturin, A.R. Akhmatkhanov, D.S. Chezganov, A.A. Esin. Appl. Phys. Lett. 103, 102905 (2013). DOI: $10.1063 / 1.4820351$.

[16] J. Schwesyg, M. Falk, C. Phillips, D. Jundt, K. Buse, M. Feier. J. Opt. Soc. Am. B. 8, 1973 (2011). DOI: $10.1364 /$ JOSAB.28.001973.

[17] A.A. Esin, A.R. Akhmatkhanov, V.Ya. Shur. Ferroelectrics 496, 102 (2016). DOI: 10.1080/00150193.2016.1157438.

[18] A.V. Yatsenko, S.V. Evdokimov, M.N. Palatnikov, N.V. Sidorov. Phys. Solid State 61, 7, 1211 (2019). DOI: 10.1134/S106378341907031X

[19] S. Klauer, M. Wöhlecke, S. Kapphan. Phys. Rev. 45, 2786 (1992). DOI: 10.1103/physrevb.45.2786.

[20] Y. Watanab, T. Sota, K. Suzuki, N. Iyi, K. Kitamura, S. Kimura. J. Phys.: Condens. Matter. 7, 3627 (1995). DOI: 10.1088/0953-8984/7/18/025.

[21] N.V. Sidorov, M.N. Palatnikov, L.A. Bobreva, N.N. Novikova. Inorg. Mater. 53, 7, 713 (2017). DOI: $10.1134 / \mathrm{S} 0020168517070172$

[22] N.A. Teplyakova, N.V. Sidorov, M.N. Palatnikov. Opt. Spectroscopy 128, 8, 1131 (2020). DOI: $10.1134 / \mathrm{S} 0030400 \mathrm{X} 20080378$

[23] Y. Furukawa, K. Kitamura, S. Takekawa, K. Niwa, Y. Yajima, N. Iyi, I. Mnushkina, P. Guggenheim, J.M. Martin. J. Cryst. Growth. 211, 230 (2000). DOI: 10.1016/S0022-0248(99)00794-0.

[24] A.V. Yatsenko, S.V. Yevdokimov, A.A. Yatsenko. Ferroelectrics 576, 157 (2021). DOI: 10.1080/00150193.2021.1888274

[25] B. Ruprecht, J. Rahn, H. Shmidt, P.Heitjans. J. Phys. Chem. 226, 431 (2012). DOI: 10.1524/zpch.2012.0226.

[26] A.V. Yatsenko, S.V. Evdokimov. Phys. Solid State 62, 3, 485 (2020). DOI: 10.1134/S1063783420030269.

[27] L. Kovács, K. Polgár, R. Capelletti, C. Mora Phys. Status Solidi A 120, 97 (1990). DOI: 10.1002/pssa.2211200107.

[28] A.V. Yatsenko, S.V. Yevdokimov, D.Yu. Sugak, I.M. Solskii. Functional Mater. 21, 1, 31 (2014).

DOI: $10.15407 / \mathrm{fm} 21.01 .031$.

Редактор Т.Н. Василевская 